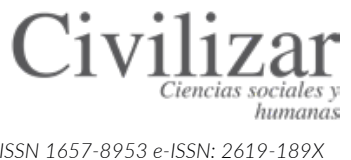

ISSN 1657-8953 e-ISSN: 2619-189X

\title{
Violencia cara a cara (offline) y en línea (online) en el noviazgo de adolescentes mexicanos ${ }^{*}$
}

\author{
Dating violence and cyber dating violence \\ in the Mexican teenage couple \\ José Luis González Ortegas \\ Christian Alexis Romero-Méndez \\ José Luis Rojas-Solís" \\ Vicente Arturo López Cortés ${ }^{\S}$
}

* Artículo de investigación

${ }^{\S}$ Licenciado en Psicología por la Benemérita Universidad Autónoma de Puebla (México).

Correo electrónico:

defler73@yahoo.com.mx

(iD) $0000-0002-6517-4314$

‡Estudiante de la licenciatura en Psicología en la Universidad del Valle de Puebla (México) y becario del Programa XXIX Verano de la Investigación Científica 2019, Academia Mexicana de Ciencias. Correo-electrónico:

christianr.mendez97@gmail.com

(iD) $0000-0003-4851-7116$

" Doctor en Psicología por la Universidad de Salamanca (España) y Profesor-investigador en la Benemérita Universidad Autónoma de Puebla (México).

Correo electrónico:

jlrojassolis@gmail.com

(iD) $0000-0001-6339-4607$

\& Doctor en Psicología por la Universidad de Salamanca (España) y Profesor-investigador en la Benemérita Universidad Autónoma de Puebla (México).

Correo electrónico:

vicente.lopez@correo.buap.mx

(iD) $0000-0003-0888-3285$

Cómo citar:

González Ortega, J. L., Romero-Méndez,

C. A., Rojas-Solís, J. L., y López Cortés,

V. A. (2020). Violencia cara a cara (offli-

ne) y en línea (online) en el noviazgo

de adolescentes mexicanos. Civilizar:

Ciencias Sociales y Humanas, 20(38),

65-80. https://doi.org/10.22518/jour. ccsh/2020.1aog

Recibido: 18/02/2020

\section{Resumen}

La violencia en el noviazgo que se ejerce a través de los medios tecnológicos y redes sociales (online) ha mostrado tener una alta frecuencia en la población de jóvenes y adolescentes. Se trata de un fenómeno relativamente nuevo cuya existencia ha sido catalogada como una extensión de la violencia ejercida cara a cara u offline. Pese a esto, son pocas las investigaciones encaminados en estudiar la relación entre ambos fenómenos. Por ello, el presente estudio tuvo como objetivo analizar la prevalencia y asociación entre la violencia offline y online en el noviazgo. Participaron 443 adolescentes (231 mujeres y 212 varones), quienes respondieron el Conflict in Adolescent Dating Relationship Inventory y el Cyber Dating Abuse Questionaire. Los resultados muestran la naturaleza bidireccional de las conductas violentas offline y online, lo que podría indicar su normalización. Entre otros datos, se resalta que la mayor perpetración de violencia emocional y física es ejercida por las mujeres, lo que iría en contravía de los perfiles convencionales de mujeres víctimas y hombres victimarios. No obstante, es preciso señalar que la violencia sexual fue mayormente ejercida por varones, lo cual es congruente con lo hallado en diversos estudios. Por último, se halló una asociación positiva entre la perpetración de la violencia offline y online en el noviazgo, lo que comprueba que las conductas violentas que se ejercen cara a cara se podrían trasladar a los medios tecnológicos y redes sociales.

\section{Palabras clave}

Violencia en el noviazgo, ciberviolencia, pareja, adolescentes, mexicanos.

\section{Abstract}

Dating violence through technological media and social networks has been shown to have a high frequency in the youth and adolescent population. It is a relatively new phenomenon whose existence has been classified as an extension of violence exercised face to face. Despite this, there is little research aimed at studying the relationship between the two phenomena. Therefore, this study aimed to analyze the prevalence and association between cyber dating violence (online) and dating violence (offline). It involved 443 adolescents (231 women and 212 men), who answered the Conflict in Adolescent Dating Relationship Inventory and the Cyber Dating Abuse Questionnaire. The results showed the two-way nature of cyber dating violence and dating violence, which could indicate their normalization. Among other data, it is highlighted that the greatest perpetration of emotional and physical violence is exercised by women, which would go against the conventional profiles of female victims and male offenders. However, it should be noted that sexual violence was mostly perpetrated by men, which is consistent with what has been found in various studies. Finally, a positive association was found between offline and online perpetration of violence in dating, which suggests that violent behaviors that are exercised face to face could be transferred to technological media and social networks.

\section{Keywords:}

Dating violence, cyber dating violence, couple, adolescent, Mexican. 


\section{Introducción}

La incorporación de los medios tecnológicos y redes sociales a la vida cotidiana ha sido una de principales transformaciones de las sociedades actuales (Livingstone y Smith, 2014). En ese sentido, se ha demostrado que sus principales implicaciones se pueden hallar en las nuevas formas de establecer, mantener, consolidar e interactuar en las relaciones interpersonales (Donoso et al., 2018; Espinoza y Rodríguez, 2017; Liu et al., 2013).

Se trata de influencias que se pueden observar también en las relaciones de noviazgo de los y las adolescentes porque la comunicación que establecen los miembros de las diadas ya no se encuentra limitada al contexto cara a cara (offline), pues se extiende al espacio virtual (online). Esta dinámica evidencia que las tecnologías y redes sociales fungen un rol importante para el establecimiento y conservación de una relación (Rodríguez y Rodríguez, 2016).

Ahora bien, la interacción y calidad de las relaciones de noviazgo en los adolescentes se puede ver afectada por la presencia de conductas violentas. Al respecto, los medios tecnológicos y redes sociales no solamente han permeado la forma de interactuar de los miembros de la diada, sino que su uso se ha visto empleado para el ejercicio de conductas indeseables dirigidas a dañar la integridad de un miembro de la pareja (Livingstone y Smith, 2014; Rodríguez y Rodríguez, 2016). Es así como ante la aparición de estas nuevas formas de violencia, diversos autores sugieren que el análisis de la violencia en el noviazgo debe ser adaptado a las nuevas condiciones de jóvenes y adolescentes - producto de los cambios sociales- (Borrajo y Gámez-Guadix, 2015; Jaen-Cortés et al., 2017), a través de la integración de nuevos conceptos como ciberviolencia de pareja o violencia online en el noviazgo.

\section{Violencia offline en el noviazgo}

La violencia offline o cara a cara en el noviazgo de adolescentes ha sido un fenómeno ampliamente estudiado en las últimas décadas debido a las graves consecuencias que acarrea. Actualmente, existen dos grandes perspectivas que analizan este fenómeno. Por una parte, un enfoque unidireccional de la violencia en el que se prioriza el estudio de la perpetración masculina y la victimización femenina, en muestras heterosexuales y atendiendo a aspectos de género (Ferrer-Pérez y Bosch-Fiol, 2019), y que se encuentra fundamento en la Teoría feminista (Muñoz et al.,
2015). De otro lado, la perspectiva bidireccional, la cual estudia la violencia de pareja sin asignar roles rígidos o permanentes. Así, este enfoque realiza un análisis más inclusivo de género en la perpetración o victimización de la violencia que, para ello, incluye aspectos individuales, familiares, sociales o culturales (Dixon y Graham-Kevan, 2011). Entre las principales manifestaciones de la violencia cara a cara se halla la práctica sistematizada de agresiones físicas, psicológicas y sexuales que ejerce un miembro de la pareja hacia otro con la finalidad de ocasionarle daño (Rey-Anacona, 2008), donde ambos miembros de la diada no se encuentran unidos por un matrimonio consolidado o un vínculo legal (Vizcarra et al., 2013). Es así como diversas investigaciones han permitido identificar algunos subtipos del fenómeno. Por un lado, se encuentra la violencia psicológica, la cual suele ser más frecuente y con mayor prevalencia en las relaciones de noviazgo (Alegría y Rodríguez, 2015; Jennings et al., 2017). Este tipo de violencia se caracteriza por el ejercicio de agresiones sutiles que pasan fácilmente desapercibidas por la víctima y que llegan a ser, incluso, normalizadas. (Hirigoyen, 2005). Se trata de actos violentos que son de carácter verbal cuya finalidad es manipular a uno de los integrantes de la diada (Flores-Garrido y Barreto-Ávila, 2018); dentro de estos comportamientos se encuentran el exigir, criticar, insultar y controlar, causando así repercusiones en el ámbito emocional (Peña et al., 2013; Wincentak et al., 2016).

Otro tipo de violencia es la violencia física. Por las consecuencias que genera, esta clase de violencia es considerada grave en tanto se ejerce con la finalidad de dañar la integridad física de la otra persona (Alegría y Rodríguez, 2015). Como medio de manifestación, se encuentran los empujones, pellizcos, bofetadas, patadas, lesiones internas y, en algunos casos, se puede llegar al homicidio (Leen et al., 2013; Olvera et al., 2012).

Por último, está la violencia sexual, de la cual se ha señalado que su perpetración es más frecuente en hombres y cuya victimización es mayor en mujeres (Rubio-Garay et al., 2017). Los actos violentos que involucra pueden ir desde el acoso sexual - que se ejerce de manera verbal, como los "piropos" o "albures" -, hasta el acto de violación, cuya forma de penalización involucra cuestiones legales (Moreno-Cubillos et al., 2007), pues atenta contra la voluntad de la persona para ejercer su sexualidad y reproductividad (Peña et al., 2013). 
En México, la prevalencia de la violencia en el noviazgo hacia las mujeres es alta. Flores-Garrido y Barreto-Ávila (2018) hallaron que la victimización de la violencia psicológica se encontraba en un $73 \%$, mientras que la violencia física en un $29.1 \%$ y la violencia sexual en un $27.7 \%$. Por otra parte, estudios realizados con muestra de varones -como el de Zamora-Damián et al. (2018) - han encontrado que la prevalencia de las conductas severas, como la violencia física y sexual, oscila entre un 9.2\% y un $20 \%$, y la violencia indirecta o psicológica, entre un $16.9 \%$ a un $36.6 \%$.

Dada la magnitud de esas cifras, es entendible que la violencia en el noviazgo esté captando la atención de la comunidad científica. Particularmente, el noviazgo en adolescentes ha resultado más atractivo debido a que es en la adolescencia cuando se aprenden actitudes y conductas que pueden ser replicadas en la edad adulta (Barón y Hernández, 2013; López-Cepero et al., 2015; Redondo et al., 2017), o trasladadas a otras relaciones, como las maritales (Peña et al., 2013; Valenzuela-Varela y Vega-López, 2015). Este tipo de comportamientos, sumado a su alta frecuencia, es lo que ha dado a la violencia en el noviazgo como resultado su carácter de problema social y salud pública (Fernández-González et al., 2013) y, a su vez, ha motivado la implementación de programas preventivos y correctivos principalmente en las instituciones educativas (Hernando-Gómez et al., 2016; Valdivia y González, 2014).

En concordancia con lo anterior, vale la pena mencionar que, desde los primeros estudios sobre la problemática, la mayoría de las investigaciones se enfocaron principalmente en la victimización femenina. Empero, hallazgos recientes sugieren la naturaleza bidireccional de las conductas violentas; es decir, tanto hombres como mujeres son víctimas y perpetradores de violencia dentro de sus relaciones (Alegría y Rodríguez, 2015; Archer, 2000). En ese sentido, se pueden encontrar algunos estudios que sustentan la victimización de los varones dentro de las relaciones de noviazgo heterosexuales (Fernández et al., 2016; Guzmán et al., 2016; Pacheco Maldonado y Castañeda Figueroa, 2013) y sugieren que las mujeres tienen la misma probabilidad de cometer violencia hacia los hombres (Nicholls y Dutton, 2008).

Añadido a ello -tal vez como resultado de los cambios sociales-, la incorporación y recono- cimiento de nuevos tipos de relaciones, como las parejas del mismo sexo, se amplió la realización de estudios. Nuevas investigaciones han permitido demostrar que la violencia no solamente es exclusiva de parejas heterosexuales, sino que su prevalencia se encuentra de igual manera en parejas homosexuales (Saldivia et al., 2017). Por otra parte, datos recientes han sugerido la manifestación de la violencia a través de los medios tecnológicos y redes sociales. Debido a lo cual, se han evidenciado nuevas formas de violencia en las relaciones de noviazgo (Gámez-Guadix et al., 2018; Rodríguez y Rodríguez, 2016).

\section{Violencia online en el noviazgo}

El surgimiento de los medios tecnológicos y redes sociales han traído consigo importantes ventajas que han facilitado el desarrollo del ser humano en la mayoría de sus ámbitos (Flach y Deslandes, 2017). Sin embargo, en contraparte, el uso inadecuado de estas herramientas por las generaciones actuales ha propiciado la aparición de nuevas problemáticas (Barquero y Calderón, 2016; Draucker y Martsolf, 2010; Machimbarrena et al., 2018). Dentro de ellas, se encuentran, por ejemplo, el ciberbullying: un fenómeno que se define como el uso de las Tecnologías de la Información y la Comunicación (redes sociales, blogs, teléfono móvil o cámaras) para el ejercicio de comportamientos de acoso (Beale y Hall, 2007) y cuya prevalencia suele afectar al ámbito educativo (Cerezo-Ramírez, 2012). De igual manera, se encuentra el sexting, entendido como todo acto de enviar fotografías y vídeos con contenido sexual a través del teléfono móvil o medios electrónicos (Mercado et al., 2016); su ejercicio se ha asociado a una nueva forma de los y las adolescentes para expresar su sexualidad (Fajardo et al., 2013). En el mismo orden de ideas, recientemente se ha investigado sobre la ciberviolencia o la violencia online en el noviazgo, la cual hace alusión al uso de las tecnologías y redes sociales como medios para el ejercicio de conductas violentas dentro de las relaciones de pareja en jóvenes (Reed et al., 2017; Rodríguez-Domínguez et al., 2018; Zweig et al., 2013). Entre sus principales características se haya su ejercicio como un medio de control e intimidación hacia la pareja o expareja con la intención de humillar, amenazar, insultar y acosar (Jaen-Cortés et al., 2017; Martín Montilla et al., 2016; Muñiz et al., 2015). El ejercicio de la violencia online en el noviazgo se ha identificado como 
un resultado de las conductas de control, producto de los sentimientos de celos manifestados por uno de los miembros de la diada hacia el otro (Afanador y Caballero, 2012; Baker y Carreño, 2016; Blanco, 2014; Borrajo et al., 2015; Hinduja y Patchin, 2020). Entre las conductas más frecuentes se hallan: pedir y compartir contraseñas de redes sociales, vigilar la actividad de la pareja, llamar varias veces al día, controlar las amistades y, hasta, presión para mantener relaciones sexuales (Flach y Deslandes, 2017; Jaen-Cortés et al., 2017; Machimbarrena et al., 2018; Muñiz et al., 2015; Van Ouytsel et al., 2017).

Estas dinámicas evidencian que el ejercicio de las conductas violentas online entre las parejas parece tener una naturaleza bidireccional de la misma manera que las conductas de violencia offline (Benavides-Delgado, 2015; Zweig et al., 2013). Sin embargo, la violencia online en el noviazgo ha mostrado tener una alta frecuencia y un carácter dominante en comparación a la violencia de pareja ejercida cara a cara (Lucio-López y Prieto-Quezada, 2014; Temple et al., 2016). La mayor accesibilidad de los adolescentes a los medios tecnológicos, redes sociales, así como al uso del ciberespacio, facilita aún más el ejercicio de las conductas violentas dentro de sus relaciones (Borrajo et al., 2015; Hinduja y Patchin, 2020; Rodríguez y Rodríguez, 2016). Así, estudios sugieren que la prevalencia de la violencia online se encuentra asociada como una extensión o nueva expresión de la violencia offline en el noviazgo (Flach y Deslandes, 2017) debido a que los actos violentos ejercidos de manera presencial se trasladan a los medios tecnológicos y redes sociales (Reed et al., 2018; Van et al., 2017). Pese a ello, aún son escasos los estudios encaminados a analizar la asociación entre estos dos fenómenos (Hinduja y Patchin, 2020; Temple et al., 2016; Zweig et al., 2014).

Aunque se ha sugerido que ser víctima de violencia offline en el noviazgo estaría relacionado con la perpetración (Caridade et al., 2019) o victimización (Cava et al., 2020) de la violencia online en el noviazgo, el estudio de esta coocurrencia de violencias sigue siendo sugerido por diversos autores debido a que el uso de los medios tecnológicos ha traído, a la par, el aumento de nuevas manifestaciones de conductas violentas en las relaciones de noviazgo. Investigaciones realizadas en México han permitido demostrar la existencia del fenómeno e identificar algunas de sus características. Se ha demostrado que los adolescentes varones pueden ser tanto víctimas como perpetradores de agresiones directas y conductas de control, esta última caracterizada por la utilización de las tecnologías y redes sociales con la intención de saber dónde y con quién se encuentra su pareja (Romo-Tobón et al., 2020). Al respecto, se sugiere que estas nuevas dinámicas de violencia deberían estar contempladas al momento de implementar los programas de intervención de violencia en parejas de jóvenes (Dick et al., 2014; Flach y Deslandes, 2017; Smith et al., 2015; Wolford-Clevenger et al., 2016).

\section{El presente estudio}

Como objetivo principal del presente estudio se plantea explorar y analizar las conductas violentas que se ejercen y victimizan cara a cara (offline) y a través de los medios tecnológicos y redes sociales (online) en las relaciones de noviazgo de los adolescentes, con el fin de determinar si existen diferencias entre varones y mujeres y si existe una asociación entre la violencia offline y online en el noviazgo.

En este sentido, se esperaría que las conductas de violencia offline y online en el noviazgo sean bidireccionales, debido a que adolescentes, tanto varones como mujeres, en algún momento han sido víctimas y perpetradores de violencia dentro de sus relaciones a través de estos medios (Benavides-Delgado, 2015). Asimismo, se esperaría encontrar que las mujeres tengan mayor victimización para la violencia offline en el noviazgo (López-Cepero et al., 2015), y los hombres, una mayor victimización en la violencia online en sus relaciones (Kellerman et al., 2013). Por último, se supone una correlación positiva entre la perpetración de la violencia offline y online en el noviazgo (Flach y Deslandes, 2017).

\section{Método}

Para llevar a cabo la consecución del objetivo planteado, se empleó un enfoque cuantitativo, con un diseño no experimental, de corte transversal y ex post facto, con alcances exploratorios, descriptivos y correlacionales.

\section{Muestra}

La muestra fue seleccionada de manera no probabilística y por conveniencia. Participaron 443 adolescentes estudiantes de bachillerato del estado 
de Puebla (México), de los cuales 231 (52.1\%) eran mujeres y $212(47.9 \%)$ varones. Fueron cuatro los criterios de inclusión para ser partícipes de la investigación: 1) ser adolescentes heterosexuales; 2) indicar tener o haber tenido una relación de pareja con al menos un mes de duración; 3) estar en edades entre los 14 y 19 años ( $\mathrm{X}=16.22 ; D T=.726)$, y 4 ) ser alumnos activos e inscritos en la institución educativa. Una mayor descripción de la muestra se podrá observar en la Tabla 1.

Tabla 1

Características sociodemográficas de la muestra

\begin{tabular}{|c|c|c|c|c|c|c|c|c|c|c|c|c|}
\hline \multicolumn{7}{|c|}{ Mujeres } & \multicolumn{6}{|c|}{ Hombres } \\
\hline & $F$ & $\bar{x}$ & Md & DT & Mín & Máx & $F$ & $\bar{X}$ & Md & DT & Mín & Máx \\
\hline \multicolumn{13}{|l|}{ Edad } \\
\hline Total & 231 & 16 & 16 & 0.72 & 15 & 19 & 212 & 16 & 16 & 0.73 & 14 & 18 \\
\hline 14 & 0 & & & & & & 1 & & & & & \\
\hline 15 & 32 & & & & & & 25 & & & & & \\
\hline 16 & 131 & & & & & & 112 & & & & & \\
\hline 17 & 62 & & & & & & 66 & & & & & \\
\hline 18 & 4 & & & & & & 8 & & & & & \\
\hline 19 & 2 & & & & & & 0 & & & & & \\
\hline \multicolumn{13}{|c|}{$\begin{array}{c}\text { Duración de la relación } \\
\text { (meses) }\end{array}$} \\
\hline Actual & - & 8.3 & 5 & 8.7 & 1 & 48 & - & 8.0 & 5 & 8.6 & 1 & 46 \\
\hline Pasada & - & 7.2 & 4 & 7.7 & 1 & 43 & - & 6.3 & 5 & 5.7 & 1 & 36 \\
\hline
\end{tabular}

Notas. F=Frecuencia, $\bar{X}=$ Media, Md=Mediana, DT=Desviación típica, Mín=Mínimo, Máx=Máximo. Fuente: Elaboración propia.

Es preciso señalar que en el análisis no se incluyeron adolescentes homosexuales debido al poco número registrado y porque el tratamiento y análisis teórico del fenómeno de la violencia en esta población superaría los objetivos del presente estudio.

\section{Instrumentos}

Cuestionario sociodemográfico. Se construyó un cuestionario con seis ítems de opción múltiple que recababan información sobre la edad, sexo, tener o haber tenido una relación de noviazgo, edad de la primera relación, tipo de relación (heterosexual u homosexual) y duración en meses de la relación.

Violencia offline en el noviazgo. Se utilizó la herramienta Conflict in Adolescent Dating Relationship Inventory (CADRI) (Hokoda et al., 2006) para evaluar la frecuencia en la que ocurren las conductas violentas de forma presencial (offline) en las relaciones de noviazgo en las naturalezas cometida y sufrida. En esta prueba, los reactivos se evalúan por medio de una escala Likert que va del $\mathrm{o}$ al 3, donde: $\mathrm{o}=$ Nunca (no ha pasado en la relación); 1=Rara vez (únicamente ha sucedido en una o dos ocasiones); $2=\mathrm{A}$ veces (ha ocurrido entre tres o cinco ocasiones); y $3=$ Con frecuencia (se ha dado en seis o más ocasiones). Asimismo, la herramienta se compone de cinco factores: violencia sexual, verbal-emocional, física, relacional y amenazas; sin embargo, para fines del presente estudio los factores de amenazas y violencia relacional fueron omitidos debido a los bajos niveles de fiabilidad obtenidos en el alfa de Cronbach. Ejemplo de los ítems incluidos en la escala de la violencia psicológica sufrida es el enunciado "Hizo algo para ponerme celoso/a", y de la cometida: "Hice algo para poner a mi chico/a celoso/a”. En la violencia física sufrida se pueden encontrar ítems como "Me 
lanzó algún objeto" y cometida: "Le lancé algún objeto". Para la violencia sexual sufrida se encuentran reactivos como "Me forzó a practicar alguna actividad sexual cuando yo no quería” y para la cometida: "Le forcé a practicar alguna actividad sexual cuando él/ella no quería”.

Violencia online en el noviazgo. Se implementó el instrumento del Cyber Dating Abuse Questionnaire (CDAQ) (Borrajo et al., 2015), que mide la perpetración y victimización de violencia a través de los medios tecnológicos y redes sociales en las relaciones de noviazgo. La herramienta se compone de una escala Likert que va del 1 al 6, donde: $1=$ Nunca (no ha pasado en nuestra relación); $2=$ No en el último año, pero sí anteriormente; $3=$ Rara vez (ha ocurrido en una o dos ocasiones); $4=\mathrm{A}$ veces (ha ocurrido entre tres y diez veces); $5=$ Con frecuencia (ha ocurrido entre once y veinte ocasiones), y $6=$ Casi siempre (ha ocurrido más de veinte veces). Además, está integrado por dos factores: control y agresión directa. Ejemplo de los ítems que conforman la escala del control sufrido es la enucnación "Mi pareja o expareja ha utilizado las nuevas tecnológicas para controlar donde he estado y con quien", y de control cometido: "He utilizado las nuevas tecnologías para controlar donde ha estado y con quien”. En el caso de la agresión directa sufrida se hallan ítems como "Mi pareja o expareja ha difundido secretos y/o informaciones comprometidas sobre mí a través de las nuevas tecnologías", y en la agresión directa cometida se encuentran reactivos como "He difundido secretos y/o informaciones comprometidas sobre mi pareja o expareja a través de las nuevas tecnologías”.

\section{Procedimiento}

Se solicitó una junta con las autoridades de las instituciones educativas (director(a), profesorado) para explicar la naturaleza y los objetivos de la investigación. Una vez obtenida su autorización, fueron ellas las encargadas de obtener el permiso de los y las responsables de los y las estudiantes para participar en el estudio. Paso seguido, se procedió a compartir el cuestionario vía online a través de la plataforma de Google forms. Para ello, se requirió de las salas de cómputo. El tiempo estimado para ser completado fue de 15 a 20 minutos.
En cuanto a los aspectos éticos es preciso señalar que en la primera parte del cuestionario se expusieron los objetivos de la investigación, seguidos de la garantía de anonimato, voluntariedad y confidencialidad de las respuestas, según los lineamientos establecidos por la Sociedad Mexicana de Psicología (2010) para el diseño y conducción de una investigación. Previo a responder el cuestionario, se solicitó el consentimiento informado de los y las participantes; se enfatizó en el carácter voluntario, anónimo y confidencial de su participación a través de un ítem ineludible que otorgaba la opción de continuar con el cuestionario o, por el contrario, abandonarlo sin penalización o pregunta alguna.

\section{Análisis de datos}

Para los análisis estadísticos se utilizó el programa de SPSS (Statistical Package for the Social Sciences) en su versión 21 para Windows. En primera instancia, se realizaron los análisis de consistencia interna de las subescalas implementadas, por medio del índice de Alpha de Cronbach. Consecutivamente, se obtuvieron las principales medidas de tendencia central para cada una de las dimensiones. De igual manera, se realizaron los análisis de normalidad a través del test de Kolmogorov-Smirnov, los cuales indicaron la distribución anormal de los datos, por lo que se optó por la utilización de técnicas no paramétricas en la realización de los análisis inferenciales. En el caso de las diferencias por sexo, se utilizó la técnica de $U$ de Mann-Whitney, y las correlaciones se obtuvieron a través del análisis de Rho Spearman.

\section{Resultados}

\section{Pruebas de fiabilidad y normalidad}

Con el objetivo de conocer la fiabilidad de las escalas implementadas y la normalidad de la distribución de los datos, se utilizaron las pruebas de alfa de Cronbach y la prueba de Kolgomorov Smirnov respectivamente. Como es posible observar en la Tabla 2, los resultados del alfa de Cronbach van entre 0.506 y 0.960, siendo la subescala de la violencia sexual la que arrojó menor consistencia interna. Sin embargo, el resto de las subescalas muestran un nivel de fiabilidad bueno. En el caso de los resultados del test de Kolgomorov Smirnov, la interpretación de los resultados indica que la distribución de los datos es distinta a la normal. 
Tabla 2

Análisis de fiabilidad y normalidad por factor, total y segmentada por sexo

\begin{tabular}{|c|c|c|c|c|c|c|}
\hline Instrumento & \multicolumn{2}{|c|}{ Violencia } & $\alpha$ Total & $\alpha$ Mujeres & $\alpha$ Hombres & Sig. K-S \\
\hline \multirow{6}{*}{ CADRI } & \multirow{2}{*}{ Sexual } & Cometida & 0.509 & 0.513 & 0.506 & 0.000 \\
\hline & & Sufrida & 0.683 & 0.660 & 0.700 & 0.000 \\
\hline & \multirow{2}{*}{ Emocional } & Cometida & 0.770 & 0.808 & 0.684 & 0.000 \\
\hline & & Sufrida & 0.833 & 0.839 & 0.830 & 0.000 \\
\hline & \multirow{2}{*}{ Física } & Cometida & 0.706 & 0.783 & 0.960 & 0.000 \\
\hline & & Sufrida & 0.749 & 0.812 & 0.668 & 0.000 \\
\hline \multirow{4}{*}{ CDAQ } & \multirow{2}{*}{ Control } & Agresor & 0.909 & 0.924 & 0.883 & 0.000 \\
\hline & & Sufrida & 0.840 & 0.849 & 0.830 & 0.000 \\
\hline & \multirow{2}{*}{ Agresión directa } & Agresor & 0.854 & 0.788 & 0.892 & 0.000 \\
\hline & & Víctima & 0.769 & 0.768 & 0.770 & 0.000 \\
\hline
\end{tabular}

Nota. $\alpha=$ Alfa de Cronbach, Sig. $K-S=K o l m o g o r o v-S m i r n o v$.

\section{Análisis descriptivos}

\section{Medidas de tendencia central}

Una vez que se obtuvo la fiabilidad de las subescalas y la distribución de los datos, se procedió a obtener las principales medidas de tendencia central (ver Tabla 3). Las conductas con mayor frecuencia para el caso de la violencia offline en el noviazgo pertenecen a la subescala de violencia emocional en la naturaleza cometida y sufrida; mientras que en la violencia online en el noviazgo, las conductas con mayor frecuencia se hallan en la subescala de la agresión directa en el rol de agresor y víctima.

Tabla 3

Medidas de tendencia central para la violencia offline y online en el noviazgo

\begin{tabular}{|c|c|c|c|c|c|c|c|c|}
\hline Instrumento & \multicolumn{2}{|c|}{ Violencia } & $N$ & $\bar{X}$ & Md & DT & Mín & Máx \\
\hline \multirow{6}{*}{ CADRI } & \multirow{2}{*}{ Sexual } & Cometida & 443 & 0.48 & 0.00 & 1.158 & 0 & 9 \\
\hline & & Sufrida & 443 & 0.69 & 0.00 & 1.551 & 0 & 12 \\
\hline & \multirow{2}{*}{ Emocional } & Cometida & 443 & 2.82 & 2.00 & 3.200 & 0 & 24 \\
\hline & & Sufrida & 443 & 3.90 & 3.00 & 4.499 & 0 & 29 \\
\hline & \multirow{2}{*}{ Física } & Cometido & 443 & 0.40 & 0.00 & 1.230 & 0 & 12 \\
\hline & & Sufrida & 443 & 0.54 & 0.00 & 1.489 & 0 & 12 \\
\hline \multirow{4}{*}{ CDAQ } & \multirow{2}{*}{ Control } & Agresor & 443 & 11.58 & 9.00 & 5.810 & 9 & 49 \\
\hline & & Víctima & 443 & 10.60 & 9.00 & 3.772 & 9 & 41 \\
\hline & \multirow{2}{*}{$\begin{array}{l}\text { Agresión } \\
\text { directa }\end{array}$} & Agresor & 443 & 12.32 & 11.00 & 3.988 & 11 & 56 \\
\hline & & Víctima & 443 & 11.66 & 11.00 & 2.205 & 11 & 35 \\
\hline
\end{tabular}

Nota. $N=$ =Muestra total, $\bar{X}=$ Media, $M d=$ =Mediana, $D T=$ Desviación típica, Mín=Mínimo, Máx=Máximo.

\section{Análisis inferenciales}

\section{Diferencias por sexo}

Con la intención de comprobar el segundo objetivo planteado en el presente estudio, se realizaron las diferencias entre varones y mujeres para determinar quién comete y sufre con mayor frecuencia las conductas de violencia offline y online en el noviazgo. Esto se realizó a través de la técnica de $U$ de Mann-Whitney. Para el caso de la violencia offline en el noviazgo, se hallaron diferencias significativas, donde la violencia sexual es mayormente cometida por varones, mientras que la violencia emocional y física es mayormente cometida por mujeres (ver Tabla 4). 
José Luis González Ortega, Christian Alexis Romero-Méndez,

José Luis Rojas-Solís, Vicente Arturo López Cortés

Tabla 4

Diferencias por sexo en las subescalas de la violencia offline en el noviazgo

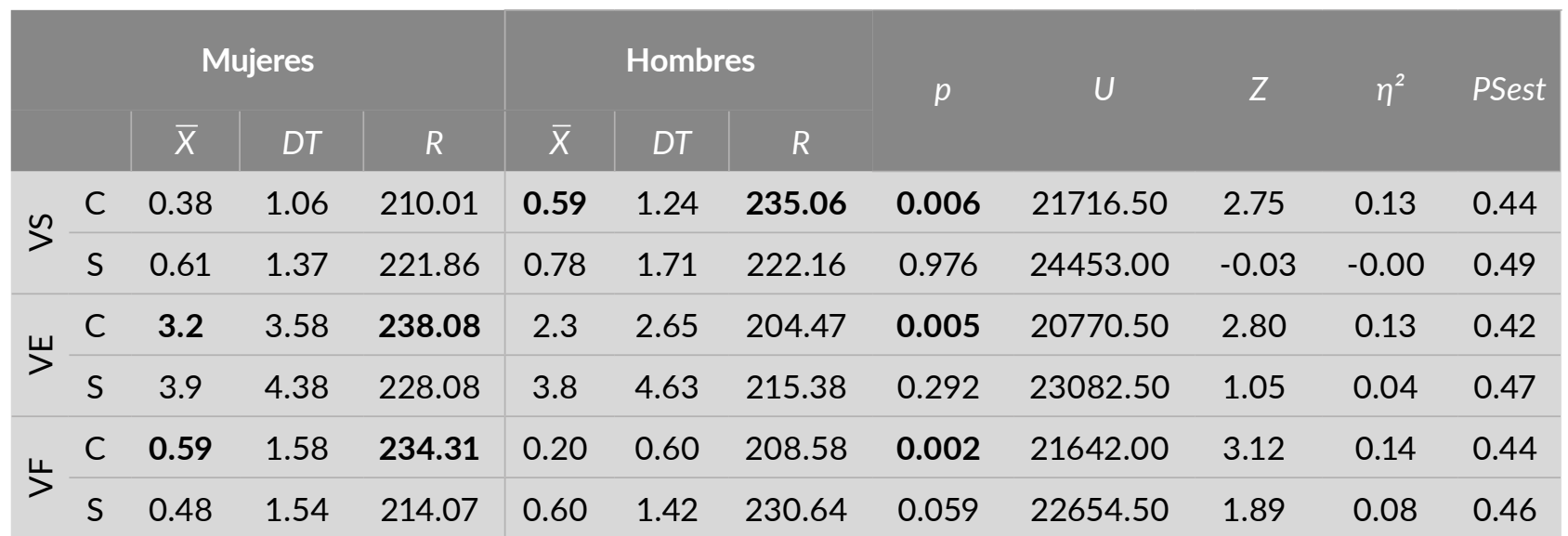

Nota. VS=Violencia sexual; VE=Violencia emocional, $V F=$ Violencia física, $C=$ Cometida, $S=S$ ufrida, $\bar{X}=$ Media, $D T=$ Desviación típica, $R=$ Rango.

Ahora bien, en la violencia online en el noviazgo (ver Tabla 5) no se hallaron diferencias estadísti- camente significativas entre varones y mujeres en ninguna de las subescalas implementadas.

Tabla 5

Diferencias por sexo en las subescalas de la violencia online en el noviazgo

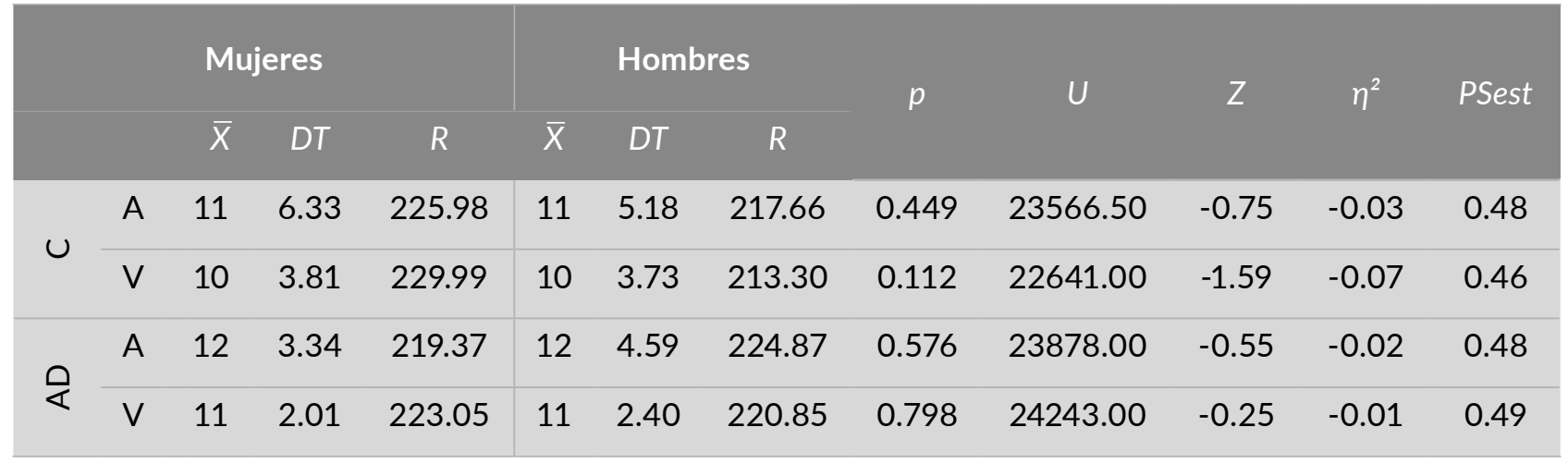

Nota. $C=$ Control, $A D=$ Agresión directa, $\boldsymbol{A}=$ Agresor, $\boldsymbol{V}=$ Víctima, $\bar{X}=$ Media, $D T=$ Desviación típica, $R=$ Rango.

\section{Asociación entre variables}

Por último, para comprobar el tercer objetivo, se realizó la asociación entre las variables estudiadas (ver Tabla 6). La obtención de los resultados se realizó por medio de análisis estadístico de Rho Spearman.

Para el caso de las mujeres, como un primer resultado, se halló una correlación moderada y significativa para la violencia sexual sufrida y cometida (rho $=0.631, p<0.00$ ). Del mismo modo, se encontró una asociación positiva moderada en la violencia verbal emocional sufrida y cometida ( $r h o=0.809$, $p<0.00$ ). Consecutivamente, se observó que existe una correlación significativa entre la violencia control en el rol de agresor con la violencia verbal emocional sufrida ( $r h o=0.520, p<0.00$ ) y con la violencia control en el rol de víctima $(r h o=0.777, p<0.00)$. Por último, se halló una relación significativa en la agresión directa en el rol de agresor con la violencia verbal emocional sufrida $(r h o=0.469, p<0.00)$ y con agresión directa en el rol de víctima $(r h o=0.556, p<0.00)$.

En la muestra de varones, en primer lugar, se muestra una asociación moderada y significativa entre la violencia sexual cometida y sufrida ( $r h o=0.678, p<0.00)$. Consecutivamente, se muestra una correlación positiva y moderada entre la violencia verbal emocional cometida y sufrida $(r h o=0.759$, $p<0.00$ ). Así también, se halló una correlación significativa en la violencia verbal emocional sufrida con la violencia control en el rol de agresor $(r h o=0.423$, $p<0.00)$ y con la agresión directa en el rol de agresor $(r h o=0.432, p<0.00)$. Por último, se observó una correlación moderada y significativa para las variables de violencia control en el rol de agresor y víctima $(r h o=0.716, p<0.00)$ y agresión directa en el rol de agresor ( $r h o=0.590, p<0.00$ ). 
Tabla 6

Asociación entre variables de la violencia offline y online en el noviazgo

\begin{tabular}{|c|c|c|c|c|c|c|c|c|c|c|}
\hline & \multicolumn{6}{|c|}{ CADRI } & \multicolumn{4}{|c|}{ CDAQ } \\
\hline & 1 & 2 & 3 & 4 & 5 & 6 & 7 & 8 & 9 & 10 \\
\hline 1 & - & $0.678^{* *}$ & $0.372^{* *}$ & $0.344^{* *}$ & $0.163^{*}$ & $0.256^{* *}$ & $0.214^{* *}$ & $0.275^{* *}$ & $0.176^{*}$ & $0.176^{*}$ \\
\hline 2 & $0.631^{* *}$ & - & $0.377^{* *}$ & $0.457^{* *}$ & $0.221^{* *}$ & $0.372^{* *}$ & $0.381^{* *}$ & $0.308^{* *}$ & $0.305^{* *}$ & $0.183^{* *}$ \\
\hline 3 & $0.322^{* *}$ & $0.343^{* *}$ & - & $0.759^{* *}$ & $0.168^{*}$ & $0.325^{* *}$ & $0.338^{* *}$ & $0.376^{* *}$ & $0.351^{* *}$ & $0.338^{* *}$ \\
\hline 4 & $0.265^{* *}$ & $0.393^{* *}$ & $0.809^{* *}$ & - & $0.253^{* *}$ & $0.499^{* *}$ & $0.423^{* *}$ & $0.313^{* *}$ & $0.432^{* *}$ & $0.297^{* *}$ \\
\hline 5 & $0.187^{* *}$ & $0.185^{* *}$ & $0.388^{* *}$ & $0.302^{* *}$ & - & $0.545^{* *}$ & $0.337^{* *}$ & $0.234^{* *}$ & $0.228^{* *}$ & $0.242^{* *}$ \\
\hline 6 & $0.146^{*}$ & $0.266^{* *}$ & $0.330^{* *}$ & $0.393^{* *}$ & $0.619^{* *}$ & - & $0.328^{* *}$ & $0.179^{* *}$ & $0.338^{* *}$ & $0.199^{* *}$ \\
\hline 7 & $0.166^{*}$ & $0.271^{* *}$ & $0.421^{* *}$ & $0.520^{* *}$ & $0.224^{* *}$ & $0.195^{* *}$ & - & $0.716^{* *}$ & $0.590^{* *}$ & $0.425^{* *}$ \\
\hline 8 & $0.206^{* *}$ & $0.281^{* *}$ & $0.421^{* *}$ & $0.433^{* *}$ & $0.194^{* *}$ & $0.196^{* *}$ & $0.777^{* *}$ & - & $0.376^{* *}$ & $0.464^{* *}$ \\
\hline 9 & $0.217^{* *}$ & $0.320^{* *}$ & $0.292^{* *}$ & $0.469^{* *}$ & $0.138^{*}$ & $0.290^{* *}$ & $0.550^{* *}$ & $0.444^{* *}$ & - & $0.584^{* *}$ \\
\hline 10 & $0.200^{* *}$ & $0.289^{* *}$ & $0.231^{* *}$ & $0.261^{* *}$ & $0.149^{*}$ & $0.265^{* *}$ & $0.343^{* *}$ & $0.355^{* *}$ & $0.556^{* *}$ & \\
\hline
\end{tabular}

Notas. Superior a la diagonal se representa las asociaciones de las variables en la muestra de varones, inferior a la diagonal se encuentra la asociación en la muestra de mujeres. $\mathbf{1}=$ Violencia sexual cometida, $\mathbf{2}=$ Violencia sexual sufrida, $\mathbf{3}=$ Violencia verbal emocional cometida, $\mathbf{4}=$ Violencia verbal emocional sufrida, $\mathbf{5}=$ Violencia física cometida, $\mathbf{6}=$ Violencia física sufrida, $\mathbf{7}=$ Control agresor, $\mathbf{8}=$ Control víctima, $\mathbf{9}=$ Agresión directa agresor, $\mathbf{1 0}=$ Agresión directa víctima.

\section{Discusión}

El objetivo del presente estudio fue explorar las conductas violentas que se ejercen y victimizan cara a cara (offline) y en línea (online) en las relaciones de noviazgo en adolescentes con el fin de determinar si la frecuencia es diferente entre varones y mujeres y establecer la posible relación entre la perpetración de la violencia offline y online en el noviazgo.

En ese sentido, los resultados obtenidos permiten aceptar la primera hipótesis, la cual refería que la violencia cara a cara y en línea en el noviazgo sería bidireccional. Como pudo observarse en los resultados, tanto hombres como mujeres en algún momento de su relación fueron víctimas, pero también perpetradores de violencia a través de estos medios (Benavides-Delgado, 2015; Zweig et al., 2013). Al respecto, se menciona que la naturaleza bidireccional de las conductas violentas repercute significativamente en la interacción de los miembros de la pareja. Por ejemplo, el intercambio recíproco de las agresiones puede mostrar un aumento progresivo, y provocar que la violencia se agudice con el tiempo (López et al., 2015), e incluso que estas conductas sean percibidas por ambos miembros de la diada como algo normal o natural dentro de su relación (Borrajo y Gámez-Guadix, 2015; Martín Montilla et al., 2016). Ello no obsta sugerir precaución en la interpretación de estos, pues las consecuencias no son igualmente graves para hombres como para mujeres.
En segundo lugar, se planteó la mayor victimización en las mujeres para la violencia offline -acorde con lo que se ha encontrado dentro de la evidencia empírica acumulada (Jenning et al., 2017)-, la cual sugiere que, como resultado de la desigualdad de poder y los estereotipos tradicionales de género, las mujeres tienden a ser mayormente víctimas de violencia en el noviazgo (Morales y Rodríguez, 2012). No obstante, los resultados del presente estudio no arrojaron diferencias significativas para la violencia sufrida; por el contrario, se halló que las mujeres indicaron cometer con mayor frecuencia dos modalidades de la violencia: emocional y física -hallazgos similares a los encontrados en otras investigaciones (Guzmán et al., 2016; Vivanco et al., 2015)-. Estos resultados sugieren la posibilidad de que exista una mayor perpetración de violencia por parte de las mujeres (Nicholls y Dutton, 2008), lo cual puede deberse a la mayor aceptación social que tiene el ejercicio de conductas agresivas de las mujeres hacia los hombres (Guzmán et al., 2016). En consecuencia, se sugiere analizar las dinámicas de la violencia fuera de los perfiles convencionales de mujeres víctimas y hombres victimarios (González y Fernández, 2014; Hernando-Gómez et al., 2016; Zamora-Damián et al., 2018).

Ahora bien, hallazgos adyacentes en el análisis de la violencia offline en el noviazgo muestran la mayor perpetración de violencia sexual por parte de los varones. Estos resultados son respaldados por 
los datos obtenidos en diversas revisiones sistemáticas y meta-analíticas que han identificado la mayor perpetración de violencia sexual por los hombres en comparación con la de las mujeres (Gracia-Leiva et al., 2019; Rubio-Garay et al., 2017; Wincentak et al., 2016). Este tipo de violencia se considera alarmante debido a que su ejercicio puede ser un factor predisponente al acto de violación (Sosa-Sánchez y Menkes-Bancet, 2015). Una explicación parte de la asimetría de poder entre hombres y mujeres ligada fuertemente a la educación tradicional, donde los varones llevan a cabo estas conductas o hazañas sexuales como una manera de reafirmar su masculinidad (Afanador y Caballero, 2012).

En el caso de la tercera hipótesis, la cual planteaba que los hombres serían mayormente víctimas de violencia online en el noviazgo, esta fue rechazada debido a que no se hallaron diferencias significativas entre varones y mujeres en ninguna de las subescalas implementadas. Los resultados son consistentes con lo encontrado por Wolford-Clevenger et al. (2016), quienes hallaron que la violencia ejercida por medios tecnológicos y redes sociales en varones y mujeres, mantienen frecuencias similares. Sin embargo, los resultados del presente estudio difieren a lo encontrado en otras investigaciones, en donde se resalta la mayor victimización de violencia online en los varones (Durán-Segura y Martínez-Pecino, 2015; Kellerman et al., 2013; Romo-Tobón et al., 2020). Dos posible explicaciones pueden encontrarse en que las mujeres tienden a ejercer también conductas de celos y control hacia los hombres, al igual que estos hacia las mujeres, y que las mujeres poseen una mayor aprobación y aceptación para el ejercicio de conductas violentas a través de los medios electrónicos (Flach y Deslandes, 2017; Van et al., 2017; Zweig et al., 2014).

En el caso de la última hipótesis, se aceptó la existencia de una correlación positiva entre la perpetración de la violencia cara a cara y en línea en el noviazgo. Los resultados sugieren que su prevalencia se debe a que los actos violentos ejercidos cara a cara podrían trasladarse a los medios tecnológicos y redes sociales (Flach y Deslandes, 2017; Reed et al., 2018; Van et al., 2017) debido a que la calidad de la interacción en las conductas online son predispuestas o determinadas por las que se tienen cara a cara (Muñiz et al., 2015). Al respecto, es pertinente mencionar que estos hallazgos constituyen un aspecto considerable en la implementación de programas de intervención dentro de las instituciones educativas, pues, ciertamente, nuevas formas de violencia en el noviazgo han eclosionado con el surgimiento de los medios tecnológicos y redes sociales (Morelli et al., 2018; Zweig et al., 2014).

\section{Conclusiones}

A manera de conclusión, el presente estudio se encaminó a explorar uno de los fenómenos que en la actualidad se ha catalogado como un problema serio de salud pública: la violencia en el noviazgo. Sin embargo, el análisis no se limitó al contexto tradicional que se ha venido estudiando en las últimas décadas -el ejercicio y recepción de la violencia de manera presencial o cara a cara-, sino que se abordó desde un perspectiva que ha surgido a la par con los cambios socioculturales debidos a la introducción de los medios tecnológicos y redes sociales, y que ha conllevado el surgimiento de nuevas formas de violencia: ciberviolencia o violencia online en el noviazgo. A este nuevo tipo de violencia, a pesar de ser un fenómeno emergente y que se encuentra en temprana exploración, se le reconoce como un problema social trascendental (Jaen-Cortés et al., 2017).

En ese sentido, se evidenció la asociación existente entre las conductas violentas ejercidas de manera presencial y las ejercidas por medios electrónicos. En ambas, se resalta su naturaleza bidireccional, lo cual sugiere que los programas de intervención no se deberían enfocar en reducir la violencia de pareja en los varones -a quienes comúnmente se les atribuye el rol de perpetradores (Smith et al., 2015)-. De igual manera, resulta pertinente ampliar el estudio y analizar su asociación con otras variables; por ejemplo, los estereotipos e ideas románticas sobre el amor (Van et al., 2017) y el ejercicio de conductas sexuales graves, como el acoso sexual y el sexting (Machimbarrena et al., 2018). En ese tenor, se resalta la necesidad de indagar cómo el fenómeno se encuentra en el contexto mexicano, debido que la literatura científica en México y América Latina es aún escasa y limitada, en comparación con otros países como Estados Unidos y de Europa.

\section{Limitaciones y futuras líneas de investigación}

Entre las limitaciones en el presente estudio se destaca la selección de la muestra, la cual fue no probabilística y por conveniencia, además de 
no representativa -aspectos que no permiten la generalización de los resultados-. La amplia franja etaria utilizada que abarcó de los 14 a los 19 años no permite unificar los resultados debido a las diferentes etapas del desarrollo en la que se encuentran los y las participantes y por tratarse de dos períodos de la adolescencia. El instrumento del CDAQ (Cyber Dating Abuse Questionaire) actualmente no se encuentra validado para la población mexicana. Del mismo modo, el factor de la violencia sexual, perteneciente al CADRI, mostró niveles de fiabilidad bajos en la muestra total y segmentada por sexos, por lo que los resultados derivados de su uso requieren cautela. Por otro lado, la evaluación solamente se aplicó a un miembro de la díada, de orientación heterosexual, y fue una muestra escolarizadas, sesgando así los resultados obtenidos.

Sin detrimento de lo anterior, es posible sugerir algunas futuras líneas de investigación en las que se incluya una muestra probabilística y representativa, se utilice una franja etaria homogénea para hacer inferencias válidas desde una perspectiva del desarrollo, se aplique la evaluación a ambos miembros de la díada, se implemente un enfoque de género inclusivo que permita integrar parejas homosexuales, así como a personas no escolarizadas, y se utilicen instrumentos validados para la población mexicana. Por otro lado, entre las posibles implicaciones del presente trabajo se destaca la importancia del estudio sobre la asociación de la violencia offline y online desde una perspectiva de género inclusiva que atienda la posibilidad de la perpetración y victimización por parte de hombres y mujeres. En ese sentido, sería importante fomentar la prevención no solo desde los factores de riesgo, sino también desde el fortalecimiento de los factores protectores en los y las adolescentes.

\section{Agradecimientos}

Al alumno Christian Alexis Romero-Méndez quien participó en la elaboración este manuscrito dentro del XXIX Verano de la Investigación Científica 2019 - Academia Mexicana de Ciencias.

\section{Referencias}

Afanador, M. I., y Caballero, M. C. (2012). La violencia sexual contra las mujeres. Un enfoque desde la criminología, la victimología y el derecho. Reflexión Política, 14(27), 122-133. Recuperado de https:// www.redalyc.org/articulo.oa?id=11023066009

Alegría, M., y Rodríguez, A. (2015). Violencia en el noviazgo: perpetración, victimización y violencia mutua. Una revisión. Actualidades en Psicología, 29(118), 57-72. doi: 10.15517/ap.v29i118.16oo8

Archer, J. (2000). Sex differences in aggression between heterosexual partners: A meta-analytic review. Psychological Bulletin, 126(5), 651-680. doi: 10.1037MJ033-2909.I26.5.651

Baker, C. K., y Carreño, P. K. (2016). Understanding the role of technology in adolescent dating and dating violence. Journal of Child and Family Studies, 25(1), 308-320. doi: 10.1007/s10826-015-0196-5

Barón, D., y Hernández, I. (2013). Violencia intrafamiliar en hogares de adolescentes pinareños con diagnóstico de trastorno disocial. Revista Ciencias Médicas, 17(2), 172-186. Recuperado de http://scielo. sld.cu/pdf/rpr/v17n2/rpr16213.pdf

Barquero, A., y Calderón, F. (2016). Influencia de las nuevas tecnologías en el desarrollo adolescente y posibles desajustes. Revista Cúpula, 3o(2), 11-25. Recuperado de https://www.binasss.sa.cr/bibliotecas/bhp/cupula/v3on2/arto2.pdf

Beale, A. V., y Hall, K. R. (2007). Cyberbullying: What school administrators (and parents) can do. The Clearing House: A Journal of Educational Strategies, Issues and Ideas, 81(1), 8-12. doi: 10.3200/ TCHS.81.1.8-12

Benavides-Delgado, J. (2015). Violencia en el noviazgo: un estudio exploratorio (Documento de trabajo No. 12). Bogotá: Ediciones Universidad Cooperativa de Colombia. doi: 10.16925/greylit1310

Blanco, M. A. (2014). Implicaciones del uso de las redes sociales en el aumento de la violencia de género en adolescentes. Comunicación y Medios, 30, 124-141. Recuperado de https://dialnet.unirioja.es/descarga/articulo/52426o2.pdf

Borrajo, E., y Gámez-Guadix, M. (2015). Comportamientos, motivos y reacciones asociadas a la victimización del abuso online en el noviazgo: un análisis cualitativo. Journal of Victimology, 2, 73-95. doi: 10.12827-rvjv-2-04

Borrajo, E., Gámez-Guadix, M., Pereda, N., y Calvete, E. (2015). The development and validation of the cyber dating abuse questionnaire among young couples. Computers in Human Behavior, 48, 358-365. doi: 10.1016/j.chb.2015.01.063

Caridade, S., Braga, T., y Borrajo, E. (2019). Cyber dating abuse (CDA): Evidence from a systematic review. Aggression and Violent Behavior, 48, 152-168. doi: 10.1016/j.avb.2019.08.018

Cava, M. J., Buelga, S., Carrascosa, L., y Ortega-Barón, J. (2020). Relations among romantic myths, offline dating violence victimization and cyber dating violence victimization in adolescents. International Journal of Environmental Research and Public Health, 17(5), 1551. doi: 10.3390/ijerph17051551 
Cerezo-Ramírez, F. (2012). Bullying a través de las TIC. Boletín Científico Sapiens- Research, 2(2), 24-29. Recuperado de https://www.srg.com.co/bcsr/index.php/bcsr/article/view/61

Dick, R. N., McCauley, H. L., Jones, K. A., Tancredi, D. J., Goldstein, S., Blackburn, S., Monasterio, E., James, L., Silverman J. G., y Miller, E. (2014). Cyber dating abuse among teens using school-based health centers. Pediatrics, 134(6), 1560-1567. doi: 10.1542/ peds.2014-0537

Dixon, L., y Graham-Kevan, N. (2011). Understanding the nature and etiology of intimate partner violence and implications for practice and policy. Clinical Psychology Review, 31(7), 1145-1155. doi: 10.1016/j. cpr.2011.07.001

Donoso, T., Rubio, M. J., y Baños, R. (2018). La adolescencia ante la violencia de género 2.0: concepciones, conductas y experiencias. Educación XXI, 21(1), 109-134. doi: 10.5944/educXX1.20180

Draucker, C. B., y Martsolf, D. (2010). The role of electronic communication technology in adolescent dating violence. Journal of child and Adolescent Psychiatric Nursing. 23(3), 133-142. doi: 10.1111/j.17446171.2010.00235. $\mathrm{x}$

Durán-Segura, M., y Martínez-Pecino, R. (2015). Ciberacoso mediante teléfono móvil e internet en las relaciones de noviazgo entre jóvenes. Comunicar, 44(22), 159-167. doi: 10.3916/C44-2015-17

Espinoza, L. A., y Rodríguez, R. (2017). El uso de tecnologías como factor del desarrollo socioafectivo en niños y jóvenes estudiantes en el noroeste de México. RICSH Revista Iberoamericana de las Ciencias Sociales y Humanísticas, 6(11). Recuperado de http:// www.redalyc.org/articulo.oa? $\mathrm{id}=503954319009$

Fajardo, M. I., Gordillo, M., y Regalado, A. B. (2013). Sexting: nuevos usos de la tecnología y la sexualidad en adolescentes. International Journal of Developmental and Educational Psychology, 1(1), 521-533. Recuperado de http://www.redalyc.org/articulo. oa? id $=349852058045$

Fernández, T., Martínez, F. A., Unzueta, C. R., y Rojas, E. (2016). Violencia hacia los hombres entre parejas jóvenes universitarias de Tijuana, México. Enseñanza e Investigación en Psicología, 21(3), 255-263. Recuperado de http://www.redalyc.org/articulo. oa?id=29248182005

Fernández-González, L., O’Leary, K. D., y MuñozRivas, M. J. (2013). We are not joking: Need for controls in reports of dating violence. Journal of Interpersonal Violence, 28(3), 602-620. doi: 10.1177/0886260512455518

Ferrer-Pérez, V. A., y Bosch-Fiol, E. (2019). El género en el análisis de la violencia contra las mujeres en la pareja: de la "ceguera” de género a la investigación específica del mismo. Anuario de Psicología Jurídica, 29(1), 69-76. doi: 10.5093/apj2019a3

Flach, R. M. D., y Deslandes, S. F. (2017). Cyber dating abuse in affective and sexual relationships: A literature review. Cadernos de Saúde Pública, 33(7), 1-18. doi: 10.1509o/0102-311Xo0138516

Flores-Garrido, N., y Barreto-Ávila, M. (2018). Violencia en el noviazgo entre estudiantes de la Universidad Nacional Autónoma de México. Un análisis mixto. Revista Iberoamericana de Educación Superior, 26(9), 42-63. Recuperado de http://www.scielo. org.mx/pdf/ries/vgn26/2007-2872-ries-9-26-42. pdf

Gámez-Guadix, M., Borrajo, E., y Calvete, E. (2018). Abuso, control y violencia en la pareja a través de internet y los smartphones: características, evaluación y prevención. Papeles de Psicólogo, 39(3), 218-227. doi: 10.23923/pap.psicol2018.2874

González, H., y Fernández, T. (2014). Hombres violentados en la pareja. Jóvenes de Baja California, México. Culturales, 2(2), 129-155. Recuperado de https:// www.redalyc.org/articulo.oa?id=69432742006

Gracia-Leiva, M., Puente-Martínez, A., Ubillos-Landa, S., y Páez-Rovira, D. (2019). La violencia en el noviazgo (VN): Una revisión de meta-análisis. Anales de Psicología, 35(2), 300-313. doi: 10.6018/analesps.35.2.333101

Guzmán, M., Contreras, V., Martínez, A., y Rojo, C. (2016). Asociación entre los estilos de apego y violencia física recibida en relaciones de noviazgo en estudiantes universitarios. Revista Argentina de Clínica Psicológica, 25(2), 177-185. Recuperado de http:// www.redalyc.org/articulo.oa?id=2819469900o8

Hernando-Gómez, A., Maraver-López, P., y Pazos-Gómez, M. (2016). Experiencias positivas y negativas en relaciones de pareja de jóvenes y adolescentes. Revista de Psicología, 25(2), 1-19. Recuperado de https://scielo.conicyt.cl/pdf/revpsicol/v25n2/ art07.pdf

Hinduja, S., y Patchin, J. W. (2020). Digital dating abuse among a national sample of U.S. Youth. Journal of Interpersonal Violence. Advance online publication. doi: 10.1177/0886260519897344

Hirigoyen, M. (2005). Mujeres maltratadas. Los mecanismos de la violencia en la pareja. Paidós

Hokoda, A., Ramos-Lira, L., Celaya, P., Vilhauer, K., Angeles, M., Ruíz, S., Malcarne, V. L., y Mora, M. (2006). Reliability of translated measures assessing dating violence among Mexican adolescents. Violence and Victims, 21(1), 117-127. doi: 10.1891/o886-6708.21.1.117

Jaen-Cortés, C. I., Rivera-Aragón, S., Reidl-Martínez, L. M., y García-Méndez, M. (2017). Violencia de pareja a través de medios electrónicos en adolescentes 
mexicanos. Acta de Investigación Psicológica, 7(1), 2593-2605. doi: 10.1016/j.aipprr.2017.01.001

Jennings, W. G., Okeem, C., Piquero, A. R., Sellers, C. S., Theolbald, D., y Farrington, D. P. (2017). Dating and intimate partner violence among young persona ages 15-30: Evidence from a systematic review. Aggression and Violent Behavior, 33, 107125. doi: 10.1016/j.avb.2017.01.007

Kellerman, I., Margolin, G., Borofsky, L., Baucom, B. R., y Iturralde, E. (2013). Electronic aggression among emerging adults: Motivations and contextual factors. Emerging Adulthood, 1(4), 293-304. doi: 10.1177/2167696813490159

Leen, E., Sorbring, E., Mawer, M., Holdsworth, E., Helsing, B., y Bowen, E. (2013). Prevalence, dynamic risk factors and the efficacy of primary interventions for adolescent dating violence: An international review. Aggression and Violent Behavior, 18(1), 159-174. doi: 10.1016/j.avb.2012.11.015

Liu, S., Yin, M., y Huang, T. (2013). Adolescents' interpersonal relationships with friends, parents, and teachers when using Facebook for interaction. Creative Education, 4(5), 335-339. https://doi. org/10.4236/ce.2013.45049

Livingstone, S., y Smith, P. K. (2014). Annual research review: Harms experienced by child users of online and mobile technologies: The nature, prevalence and management of sexual and aggressive risks in the digital age. The Journal of Child Psychology and Psychiatry, 55(6), 635-654. doi: 10.1111/jcpp.12197

López, L., Fundora, Y., Valladares, A., Ramos, Y., y Blanco, Y. (2015). Prevalencia de la violencia física y psicológica en relaciones de pareja heterosexuales. Revista Finlay, 5(3), 161-169. Recuperado de http:// scielo.sld.cu/pdf/rf/v5n3/rfo3305.pdf

López-Cepero, J., Rodríguez-Franco, L., Rodríguez-Díaz, F. J., Bringas, C., y Paíno, S. G. (2015). Percepción de la victimización en el noviazgo de adolescentes y jóvenes españoles. Revista Iberoamericana de Psicología y Salud, 6(2), 64-71. doi: 10.1016/j. rips.2015.04.001

Lucio-López, L. A., y Prieto-Quezada, M. T. (2014). Violencia en el ciberespacio en las relaciones de noviazgo adolescente. Un estudio exploratorio en estudiantes mexicanos de escuelas preparatorias. Revista de Educación y Desarrollo, 31, 61-72. Recuperado de http://www.seg.guanajuato.gob.mx/ Ceducativa/CDocumental/Doctos/2014/Octubre/ Violenciaciberespacio.pdf

Machimbarrena, J. M., Calvete, E., Fernández-González, L., Álvarez-Bardón, A., Álvarez-Fernández, L., y González-Cabrera, J. (2018). Internet risks: An overview of victimization in cyberbullying, cyber dating abuse, sexting, online grooming and problematic internet use. International Journal of Envi- ronmental Research and Public Health, 15(11), 2-15. doi: 10.3390/ijerph15112471

Martín Montilla, A., Pazos Gómez, M., Montilla Coronado, M. V. C., y Romero Oliva, C. (2016). Una modalidad actual de violencia de género en parejas de jóvenes: Las redes sociales. Educación XX1, 19(2), 405-429. doi: 10.5944/educXX1.13934

Mercado C. T., Pedraza, F. J., y Martínez, K. I. (2016). Sexting: su definición, factores de riesgo y consecuencias. Revista sobre la Infancia y la Adolescencia, (10), 1-18. doi: 10.4995/reinad.2016.3934

Morales, N. E., y Rodríguez, V. (2012). Experiencias de violencia en el noviazgo de mujeres de Puerto Rico. Revista Puertorriqueña de Psicología, 23, 579o. Recuperado de http://pepsic.bvsalud.org/pdf/ reps/v23/ao3.pdf

Morelli, M., Bianchi, D., Chirumbolo, A., y Baiocco, R. (2018). The cyber dating violence inventory. Validation of a new scale for online perpetration and victimization among dating partners. European Journal of Developmental Psychology, 15(4), 464471. doi: 10.1080/17405629.2017.1305885

Moreno-Cubillos, C. L., Osorio-Gómez, L. S., y Sepúlveda-Gallego, L. E. (2007). Violencia sexual contra las estudiantes de la Universidad de Caldas (Colombia). Estudio de corte transversal. Revista Colombiana de Obstetricia y Ginecología, 58(2), 116-123. Recuperado de https://www.redalyc.org/articulo. oa?id=19521432700 4

Muñiz, M., Cuesta, P., Monreal, M. C., y Povedano, A. (2015). Violencia de pareja online y offline en la adolescencia: el rol de la soledad y del género. $R e-$ vista sobre la Infancia y la Adolescencia, 9, 85-97. doi: 10.4995/reinad.2015.3898

Muñoz, M., González-Lozano, P., Fernández-González, L., y Fernández-Ramos, S. (2015). Violencia en el noviazgo. Realidad y prevención. Pirámide.

Nicholls, T. L., y Dutton, D. G. (2008). Abuse committed by women against male intimates. Journal of Couples Therapy, 10(1), 41-57. doi: 10.1300/ Jo36v10no1_04

Olvera, J. A., Arias, J., y Amador, R. (2012). Tipos de violencia en el noviazgo: estudiantes universitarias de la UAEM, Zumpango. Revista Electrónica de Psicología de Iztacala, 15(1), 150-171. Recuperado de http://www.revistas.unam.mx/index.php/repi/ article/view/30908

Pacheco Maldonado, K., y Castañeda Figueroa, J. G. (2013). Hombres receptores de violencia. Avances en Psicología, 21(2), 207-221. doi: 10.33539/avpsicol.2013.v21n2.287

Peña, F., Zamorano, B., Hernández, G., Hernández, M., Vargas, J. I., y Parra, V. (2013). Violencia en el noviazgo en una muestra de jóvenes mexicanos. 
Revista Costarricense de Psicología, 32(1), 27-40. Recuperado de https://dialnet.unirioja.es/descarga/articulo/4836509.pdf

Redondo, J., Inglés, C. J., y García, K. L. (2017). Papel que juega la edad en la violencia en el noviazgo de estudiantes de la Universidad Pontificia Bolivariana de Bucaramanga. Diversitas, 13(1), 41-54. Recuperado de https://www.redalyc.org/jatsRepo/679/67952833003/index.html

Reed, L. A., Tolman, R. M., y Ward, L. M. (2017). Gender matters: Experiences and consequences of digital dating abuse victimization in adolescent dating relationships. Journal of Adolescence, 59, 79-89. doi: 10.1016/j.adolescence.2017.05.015

Reed, L. A., Ward, L. M., Tolman, R. M., Lippman, J. R., y Seabrook, R. C. (2018). The association between stereotypical gender and dating beliefs and digital dating abuse perpetration in adolescent dating relationships. Journal of Interpersonal Violence. [Avance publicación] doi: 10.1177/0886260518801933

Rey-Anacona, C. A. (2008). Prevalencia, factores de riesgo y problemáticas asociadas con la violencia en el noviazgo: una revisión de la literatura. Avances en Psicología Latinoamericana, 26(2), 227-241. Recuperado de https://www.redalyc.org/articulo. oa? $\mathrm{id}=79926209$

Rodríguez, T., y Rodríguez, Z. (2016). El amor y las nuevas tecnologías: experiencias de comunicación y conflicto. Comunicación y sociedad, (25), 15-41. Recuperado de http://www.scielo.org.mx/pdf/ comso/n25/n25a2.pdf

Rodríguez-Domínguez, C., Durán Segura, M., y Martínez Pecino, R. (2018). Ciberagresores en el noviazgo adolescente y su relación con la violencia psicológica, el sexismo y los celos. Health and Addictions, 18(1), 17-27. Recuperado de https://idus. us.es/handle/11441/70532

Romo-Tobón, R. J., Vázquez-Sánchez, V., Rojas-Solís, J. L., y Alvídrez, S. (2020). Cyberbullying y Ciberviolencia de pareja en alumnado de una universidad privada mexicana. Propósitos y Representaciones, 8(2), 1-20 doi: 10.20511/pyr2020.v8n2.303

Rubio-Garay, F., López-González, M. A., Carrasco, M. A., y Amor, P. J. (2017). Prevalencia de la violencia en el noviazgo: una revisión sistemática. Papeles del Psicólogo, 38(2), 135-147. doi: 10.23923/pap.psicol2017.2831

Saldivia, C., Faúndez, B., Sotomayor, S., y Cea, F. (2017). Violencia íntima en parejas jóvenes del mismo sexo en Chile. Última Década, (46), 184-212. Recuperado de https://scielo.conicyt.cl/pdf/udecada/ v25n46/0718-2236-udecada-25-46-00184.pdf

Smith, J., Mulford, C., Latzman, N., Tharp, A., Holditch, P., y Blachman-Demner, D. (2015). Taking stock of behavioral measures of adolescent dating violence. Journal Aggress Maltreat Trauma, 24(6), 674-692. doi: 10.1080/10926771.2015.1049767

Sociedad Mexicana de Psicología (2010). Código Ético del Psicólogo. Trillas

Sosa-Sánchez, I. A., y Menkes-Bancet, C. (2015). Amarte duele. La violación sexual en las relaciones de noviazgo. Un análisis de sus determinantes sociales. Papeles de Población, (87), 43-62. Recuperado de http://www.scielo.org.mx/pdf/pp/v22n87/14057425-pp-22-87-00043.pdf

Temple, J. R., Choi, H. J., Brem, M., Wolford-Clevenger, C., Stuart, G. L., Peskin, M. F., y Elmquist, J. (2016). The temporal association between traditional and cyber dating abuse among adolescents. Journal of Youth and Adolescence, 45, 340-349. doi: 10.1007/ s10964-015-0380-3

Valdivia, M. P., y González L. A. (2014). Violencia en el noviazgo y pololeo: Una actualización proyectada hacia la adolescencia. Revista de Psicología, 32(2), 330-355. Recuperado de http://www.scielo. org.pe/scielo.php?script=sci_arttext\&amp;pi$\mathrm{d}=$ So25492472014000200006

Valenzuela-Varela, A., y Vega-López, M. G. (2015). Violencia en el noviazgo en adolescentes. Un problema de salud pública. Salud Jalisco, (3), 164-168. Recuperado de https://www.medigraphic.com/pdfs/ saljalisco/sj-2015/sj153g.pdf

Van Ouytsel, J., Ponnet, K., y Walrave, M. (2017). Cyber dating abuse: Investigating digital monitoring behaviors among adolescents from a social learning perspective. Journal of Interpersonal Violence. doi: 10.1177/0886260517719538

Vivanco, R., Espinoza, S., Romo, C., Véliz, A., y Vargas, A. (2015). Perpetración y victimización de la violencia en relaciones de parejas en jóvenes que cursan educación superior en la ciudad de Osorno, Chile. Polis, 4o, 1-17. Recuperado de http://journals.openedition.org/polis/10863

Vizcarra, M. B., Poo. A. M., y Donoso, T. (2013). Programa educativo para la prevención de la violencia en el noviazgo. Revista de Psicología, 22(1), 48-61. Recuperado de http://www.redalyc.org/articulo. oa?id=26429848007

Wincentak, K., Connolly, J., y Card, N. (2016). Teen dating violence: A meta-analytic review of Prevalence Rates. Psychology of Violence. 7(2), 224-241. doi: 10.1037/a0040194

Wolford-Clevenger, C., Zapor, H., Brasfield, H., Febres, J., Elmquist, J., Brem, M., Shorey, R. C., y Stuart, G. (2016). An examination of the partner cyber abuse questionnaire in a college student sample. Psychology Violence, 6(1), 156-162. doi: 10.1037/ aoo39442 
Zamora-Damián, G., Alvídrez-Villegas, S., Aizpitarte-Gorrochategui, A., y Rojas-Solís, J. L. (2018). Prevalencia de violencia en el noviazgo en una muestra de varones adolescentes mexicanos. Revista de Psicología y Ciencias del Comportamiento, 9(1), 30-53. doi: 10.29365/rpcc.20180529-63

Zweig, J. M., Dank, M., Yahner, J., y Lachman, P. (2013). The rate of cyber dating abuse among teens and how it relates to other forms of teen dating violence. Journal of Youth and Adolescence, 42, 10631077. doi: 10.1007/s10964-013-9922-8

Zweig, J. M., Lachman, P., Yahner, J., y Dank, M. (2014). Correlates of cyber dating abuse among teens. Journal of Youth and Adolescence, 43(8), 1306-1321. doi: 10.1007/s10964-013-0047-X 
